\title{
Nonlinear Dynamic Force Balance Mass-spring-damper Model of Laser Droplet Generation from a Metal Wire
}

\author{
Andrej Jeromen* - Edvard Govekar \\ University of Ljubljana, Faculty of Mechanical Engineering, Slovenia
}

In the laser droplet generation process a metal wire is continuously fed into the focus of a pulsed annular laser beam that melts the wire-end and forms a growing pendant droplet that is then detached from the wire. The process is highly complex due to its non-stationarity, nonlinearity, and the interplay of numerous physical phenomena. With the aim of describing the process, a low-dimensional, non-linear, dynamic force balance, mass-spring-damper model of the pendant droplet with time-dependent coefficients was formulated based on experimental observations of the process. A comparison between the modelled and experimental droplet centroid vertical position time series and their timefrequency maps showed that the model captures the essential pendant-droplet dynamics in the selected laser-pulse frequency range between $60 \mathrm{~Hz}$ and $190 \mathrm{~Hz}$. It was also found that the modelled time of detachment and the detached-droplet diameter were in good agreement with the experimental results, including the bifurcation at the laser-pulse frequency of $120 \mathrm{~Hz}$ and the coexistence of two detached-droplet diameter values below that frequency. In addition, the pendent droplet's lateral oscillation and the Rayleigh-Plateau instability were identified as having a significant influence on the process outcome in certain laser-pulse frequency ranges.

Keywords: laser droplet generation, mass-spring-damper system, nonlinear model, time series, time-frequency analysis

Highlights

- Low-dimensional model captures the essential dynamics of the droplet-generation process.

- Modelled time series and their time-frequency maps correspond well with experiments.

- Model predicts the detached droplet diameter as a function of laser-pulse frequency.

- $\quad$ Lateral oscillation and Rayleigh-Plateau instability can influence the process.

\section{O INTRODUCTION}

Direct laser metal deposition is one of the established metal-based additive manufacturing processes [1]. It usually employs input material in shape of wire [2] or powder [3]. In contrast to the wire or powder deposition process, where the laser is used to generate melt pool on the workpiece surface, the process of metal droplet deposition significantly reduces the workpiece heat load, since the laser beam is used only for droplet generation. In consequence, metal droplets have been employed in various innovative technologies, including the droplet joining of electrical contacts, and of dissimilar and temperaturesensitive materials [4], as well as 3D printing [5]. A necessary basis for droplet-based technologies is a reliable method for the generation of metal droplets. To achieve this, a number of metal-droplet generation methods based on melt ejection from a nozzle have been developed, employing different techniques to generate the ejection pulse: piezoelectric [6], pneumatic [7], solenoid [8], and electromagnetic [9]. The above methods employ a heated crucible to maintain a reservoir of molten metal. Metals with relatively low melting temperatures are used, such as solder [9], indium, lead, zinc [7], and aluminum [10]. To avoid an energy-inefficient crucible and simplify the system, a method employing induction melting of the wire tip in the pressurized tube leading to the nozzle was proposed [11]. Common to all of the above methods is the nozzle, the diameter of which should be well below the diameter of the generated droplets and is subject to wear. To avoid melt oxidation, leading to nozzle clogging, a low-oxygen-content atmosphere should be provided [7].

Besides the methods based on melt ejection, several laser-based methods have been developed that take advantage of the high level of spatial and temporal control of the laser's energy delivery. They differ in terms of the shape of the input metal material, which can be either a foil [12], a spherical preform [13], or a wire [14]. Although it achieves lower dropletgeneration frequencies compared to melt-ejection methods, the laser droplet generation from a wire using an annular laser beam [15] shows strong application potential, since it can employ metals with a high melting point, such as nickel [14], is not very sensitive to oxygen content, and the related system includes no wearable parts. In annular laser beam droplet generation (ALDG), a metal wire is fed axially into the center of the annular laser beam, which is focused on the circumference of the wire-end. ALDG can be performed either as drop-on-demand ALDG [14], where the pendant-droplet formation and detachment 
are achieved by a single time-dependent, laser-pulse synchronized with the feeding of the wire, or as continuous ALDG [15], where periodic laser pulses melt the end of the wire, which is fed continuously with a constant velocity into the focus of the annular laser beam. An experimental study of the continuous ALDG has shown that, depending on the laser-pulse frequency, the detachment of the pendant droplet is stimulated by the resonance of a droplet-oscillation eigenmode with the polar wavenumber $n=1,2$, or 3 [15]. In addition, a recent experimental study, based on time series and focused on the $n=1$ droplet-oscillation eigenmode in the laser-pulse frequency range between $60 \mathrm{~Hz}$ and $190 \mathrm{~Hz}$ [16], showed that the essential dynamics of the pendant droplet are very similar to that of a time-varied, mass-spring-damper system. In the study, the effective spring stiffness was determined as a function of the droplet volume and the resonancecaused droplet detachment was confirmed. These results indicate that despite the process being highly complex due to its non-stationarity, non-linearity, and the interplay of numerous physical phenomena, a lowdimensional model could describe the core dynamics of the process. Such a model would provide insight and an understanding of the process, and would also be beneficial for process monitoring, forecasting, and control.

Low-dimensional, mass-spring-damper models were already employed for the description of a process similar to continuous ALDG, i.e., gas metal arc welding (GMAW) in the globular mode. The structure of the employed models ranges from the simplest static force balance model that uses either a point mass and constant coefficients [17] (or also includes droplet geometry and time-dependent coefficients [18]) to a dynamic force balance model that includes the inertial force in the droplet-detachment criterion [19]. The most important coefficients of those models are the nonlinear spring stiffness and the damping coefficient, which could be theoretically determined based on the droplet's surface-energy gradient and the viscous damping, respectively. However, the resulting theoretical value of the spring stiffness was found to be too large, which is consistent with the results of the ALDG study [16]. In contrast, the theoretically determined viscous damping coefficient was reported to be much too small to match the experimental droplet dynamics, which could be caused by the rotational fluid flow inside the droplet [18]. Although the above models can provide the basis for formulating the model of continuous ALDG, they cannot be used directly, since the electric arc in GMAW exerts an electromagnetic force that is not present in ALDG as well as there being a difference in the droplet excitation, which in GMAW is achieved by modulation of the electric current, while in continuous ALDG it is achieved by melting of the wire by the laser beam.

The aim of this work is to formulate a mathematical model of the continuous ALDG process in the form of a low-dimensional, dynamic force balance, massspring-damper system with time-varied coefficients and to evaluate the formulated model by comparing the resulting time series and their time-frequency maps with the experimental ones. To achieve this, the first section briefly describes the continuous ALDG experimental system and process, as well as the relevant results of the previous experimental analysis [16]. Based on these results, in the second section the time-varied coefficients are determined and the dynamic force balance, mass-spring-damper model of droplet growth and detachment is formulated. In the third section, the model-generated time series, their time-frequency maps, and the diameters of the detached droplets are presented, discussed, and compared with the experimental results.

\section{EXPERIMENT}

A schematic presentation of the ALDG experimental system [14] is shown in Fig. 1. The system consists of a Nd:YAG pulsed-laser source, an optical part that transforms the collimated laser beam into an annular form and then focuses it, and a wire-feeding unit, which feeds the wire into the focus of the laser beam. In order to prevent oxidation, the process region is flooded by argon gas of purity 5.0 using a coaxial nozzle.

In the continuous ALDG process [15], the wire is fed at a constant feeding velocity, whereas the laser source emits periodic laser pulses at a certain preset frequency in order to melt the wire-end. Due to the periodic heating and melting of the wire directly above the pendant droplet, the droplet grows by incorporating the melted material and is at the same time excited into oscillation since its attachment point is periodically displaced.

The continuous ALDG experiments [15] were performed using a nickel wire and the values of the process parameters, summarized in Table 1. At different values of the laser-pulse frequency $f_{\mathrm{p}}$, the power of the laser pulse was adjusted to maintain a constant average laser power $P_{\text {avg }}$. The process was monitored by means of a high-speed IR sensitive camera, which captured images of the laser beam's focus region with a frequency of 1445 frames per 
second, an integration time of $40 \mu \mathrm{s}$, and an image array size of $64 \times 128$. Using the captured images, the shape and the position time dependence of the hot pendant droplet were obtained.

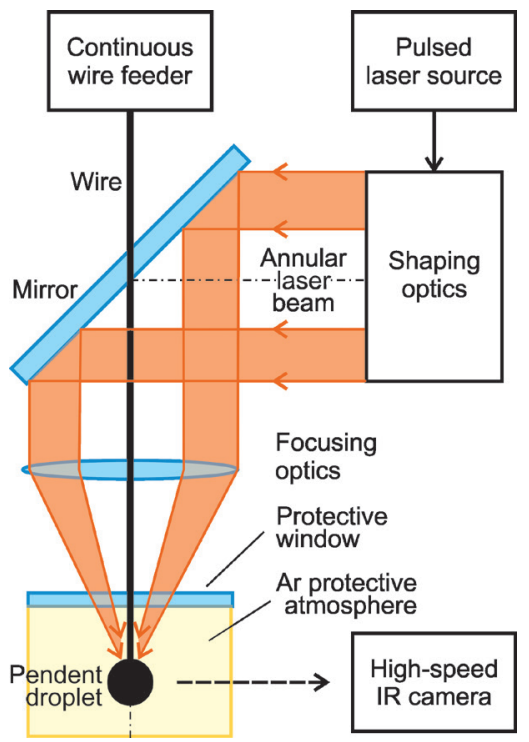

Fig. 1. Scheme of the ALDG experimental setup

Table 1. Summary of the process parameters

\begin{tabular}{lc}
\hline Parameter & Value \\
\hline Wire diameter, $d_{\mathrm{w}}$ & $0.25 \mathrm{~mm}$ \\
\hline Wire feeding velocity, $v_{\mathrm{w}}$ & $0.06 \mathrm{~m} / \mathrm{s}$ \\
\hline Laser pulse frequency, $f_{\mathrm{p}}$ & $50 \mathrm{~Hz}$ to $300 \mathrm{~Hz}$ \\
\hline Laser pulse duration, $\tau$ & $0.8 \mathrm{~ms}$ \\
\hline Average laser power, $P_{\mathrm{avg}}$ & $120 \mathrm{~W}$ \\
\hline
\end{tabular}

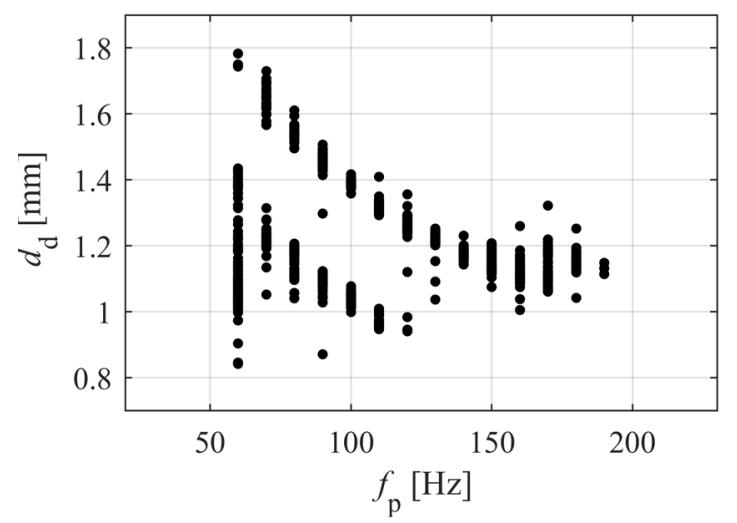

Fig. 2. Experimental detached-droplet diameter $d_{\mathrm{d}}$ as a function of the laser-pulse frequency $f_{\mathrm{p}}$

The present study is focused on the laser-pulse frequency $f_{\mathrm{p}}$ range between $60 \mathrm{~Hz}$ and $190 \mathrm{~Hz}$, where the pendant droplet's detachment is triggered by the resonance of the $n=1$ eigenmode [15]. In order to provide the reader with the experimental background that forms the starting point of the present study, the main results of the continuous ALDG experimental study [16] are reproduced and briefly commented on here. In the selected frequency range, the main dynamics of the pendant droplet could be described as that of a forced, time-variable, lightly damped, massspring oscillating system, the mass of which increases with time [16]. The effective spring constant $k$ of the system was found to show a power-law dependence on the volume $V$ of the pendant droplet:

$$
k(V)=k_{0}\left(\frac{V}{V_{0}}\right)^{a},
$$

where $k_{0}=0.011 \mathrm{~N} / \mathrm{m}, \quad V_{0}=1 \mathrm{~m}^{3}$, and $a=-0.29$, whereas the system's damping ratio could not be estimated from the experimental data [16]. The results of the experimental analysis showed that the pendant droplets detach when in resonance with the laser-pulse frequency $f_{\mathrm{p}}$. However, at frequencies $f_{\mathrm{p}}$ between $60 \mathrm{~Hz}$ and $120 \mathrm{~Hz}$ smaller droplets were also observed to detach when in resonance with the laser-pulse frequency's second multiple $2 f_{\mathrm{p}}$. In Fig. 2 , the detached droplet diameter $d_{\mathrm{d}}$ values [15] are reproduced in the selected laser-pulse frequency $f_{\mathrm{p}}$ range. The upper branch in Fig. 2 denotes the diameters of the droplets that were detached in resonance with $f_{\mathrm{p}}$, whereas the lower branch denotes the diameters of the smaller droplets that were detached in resonance with $2 f_{\mathrm{p}}$. In the frequency range $f_{\mathrm{p}}$ between $60 \mathrm{~Hz}$ and $120 \mathrm{~Hz}$ droplets belonging to both branches were generated in a seemingly random sequence.

\section{MODEL FORMULATION}

Before formulating the model, several relevant properties of the continuous ALDG process are presented. The pendant droplet, which is going to be represented by a point-mass in the model, grows and oscillates in the process. Due to its increasing mass, the properties of the droplet, such as its eigenmode frequencies, the attachment-to-droplet-diameter ratio, the effective spring constant, and the damping coefficient, are all time dependent. Additionally, due to the periodic heating and melting of the wire above the droplet, the pendant droplet's oscillation is forced by the periodic mass increases and the corresponding attachment-point displacements. It should be noted that the forcing in continuous ALDG is not harmonic, but closer to impulse-like, since the duration of the laser pulse $\tau$ is short compared to the pulse period $1 / f_{\mathrm{p}}$ between $5.3 \mathrm{~ms}$ and $17 \mathrm{~ms}$ in the selected frequency 
range. The pendant droplet can also be described by the values of the following governing dimensionless groups [20], calculated using the properties of molten nickel, summarized in Table 2, and a typical pendant-droplet diameter $d=1 \mathrm{~mm}$. The large value of the Reynolds number $\operatorname{Re}=\left(\sigma d \rho /\left(2 \mu^{2}\right)\right)^{1 / 2}=1.6 \times 10^{4}$ indicates that the influence of viscosity is small, while the small value of the gravitational Bond number is $G=\left(\rho g d^{2}\right) /(4 \sigma)=1.1 \times 10^{-2}$, where $g=9.81 \mathrm{~m} / \mathrm{s}^{2}$ is the acceleration due to gravity, indicates that the influence of surface tension is large compared to gravity, which is reflected in the observed, nearly spherical, shape of the pendant droplet.

Table 2. Summary of physical properties of molten nickel at the melting temperature [21]

\begin{tabular}{lc}
\hline Property & Value \\
\hline Temperature of melting, $T_{\mathrm{m}}$ & $1728 \mathrm{~K}$ \\
\hline Density, $\rho$ & $7905 \mathrm{~kg} / \mathrm{m}^{3}$ \\
\hline Viscosity, $\mu$ & $1.66 \times 10^{-4} \mathrm{~Pa} \cdot \mathrm{s}$ \\
\hline Surface tension, $\sigma$ & $1.778 \mathrm{~N} / \mathrm{m}$ \\
\hline Thermal diffusivity, $\alpha$ & $5.8 \times 10^{-6} \mathrm{~m}^{2} / \mathrm{s}$ \\
\hline
\end{tabular}

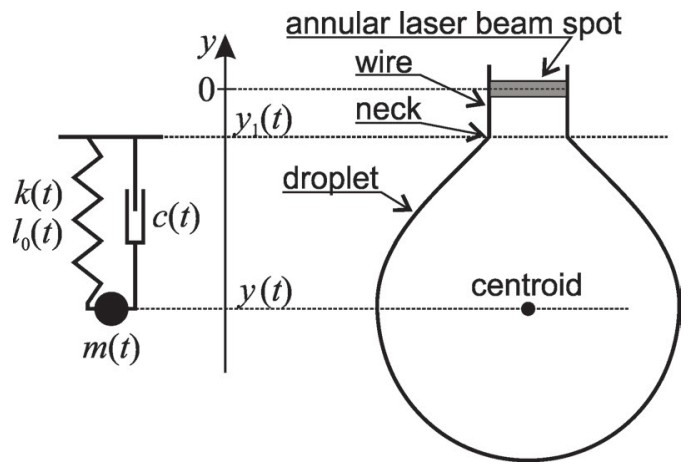

Fig. 3. Scheme of the mass-spring-damper model and its geometrical relation to the pendant droplet

In order to model the pendant droplet's centroid oscillation and detachment in continuous ALDG, a forced, time-variable, mass-spring-damper model was formulated. The scheme of the model system and its geometrical relation to the pendant droplet is presented in Fig. 3. The model system consists of a time-dependent point mass $m(t)$, which is suspended on a spring with a time-dependent spring stiffness $k(t)$ and an unloaded length $l_{0}(t)$, and a viscous damper with a time-dependent damping coefficient $c(t)$. The position $y(t)$ of the model point mass $m$ corresponds to the position of the pendant-droplet centroid, whereas the position $y_{1}(t)$ of the model spring-attachment point corresponds to the position of the pendant droplet neck, i.e., of the attachment to the wire.
The equation of motion of the point mass position $y$ was formulated as a second-order differential equation with time-varying coefficients, as follows:

$$
\begin{aligned}
m(t) \ddot{y} & =-m(t) g+k(t)\left(y_{1}(t)-y-l_{0}(t)\right)- \\
& -c(t)\left(\dot{y}_{1}-\dot{y}\right) .
\end{aligned}
$$

The terms on the right-hand side of Eq. (2) represent the gravitational force, the spring force, and the viscous damping force. The drag force on the droplet moving in the surrounding protective atmosphere of argon can be taken as negligible. For this reason the damping force was assumed to be mainly the result of the droplet's inner flows [18], and was modelled as being proportional to the relative velocity $\dot{y}_{1}-\dot{y}$ between the droplet centroid and the droplet neck, i.e., the spring attachment position. In the following text, the time-dependent coefficients of Eq. (2), the initial values of the model, and the pendant-droplet detachment condition are determined based on the experimental observations.

The displacement of the spring-attachment position $y_{1}$ is the source of the external forcing. Its time dependence $y_{1}(t)$ is a consequence of the constant-velocity wire feeding combined with the laser-induced periodic melting of the wire above the droplet neck. The function $y_{1}(t)$ was formulated as a piecewise linear function. Between the consecutive pulses, the spring-attachment position $y_{1}$ was assumed to decrease linearly with the velocity of the wire feeding $v_{\mathrm{w}}$. At the time of the laser-pulse trigger, the laser beam is directed onto the wire circumference above the pendant droplet's neck. For this reason, during a laser pulse of duration $\tau$, the position $y_{1}$ was assumed to increase linearly up to the position of the laser beam focus at $y=0$, as indicated in Fig. 3. The minimum value $y_{1 \min }$ of the spring-attachment position $y_{1}$ was thus expressed as $y_{1 \min }=-v_{\mathrm{w}}\left(t_{0}-\tau\right)$, where $v_{\mathrm{w}}$ is the downward wire-feeding velocity magnitude, $t_{0}=1 / f_{\mathrm{p}}$ denotes the time period of the laser pulses, and $\tau$ is the duration of the laser pulse. The experimentally determined minimum values of $y_{1}$ correspond well to the above-determined $y_{1 \min }$ for very small droplets, i.e., at the very beginning of their growth. However, during the time $t_{1}=0.3 \mathrm{~s}$ from the beginning of the droplet growth, the experimentally observed minimum value gradually increased to approximately half of its theoretical value $-v_{\mathrm{w}}\left(t_{0}-\tau\right)$. The observed increase of $y_{1 \min }$ can be explained by the increasing mass of the liquid pendant droplet, which contains an increased amount of energy and is increasingly able to melt the wire between the laser pulses. However, since the temperature of the droplet is close to the 
melting temperature, the melting of the wire by the pendant droplet saturates and the minimum value $y_{1 \min }$ stabilizes at half of its theoretical value for the selected values of the average laser power, the wire diameter $d_{\mathrm{w}}$, and the feeding velocity $v_{\mathrm{w}}$. In order to take into account this experimentally observed effect, the minimum value $y_{1 \min }$ was defined as time dependent:

$$
y_{1 \min }(t)=\left\{\begin{array}{c}
-v_{\mathrm{w}}\left(t_{0}-\tau\right)\left(1-\frac{t}{2 t_{1}}\right) ; \quad t<t_{1}, \\
-\frac{v_{\mathrm{w}}\left(t_{0}-\tau\right)}{2} ; \quad t \geq t_{1} .
\end{array}\right.
$$

Based on this, the spring-attachment position's time dependence $y_{1}(t)$ was then expressed as:

$$
\begin{aligned}
& y_{1}(t)= \\
& =\left\{\begin{array}{c}
y_{1 \min }(t) \frac{t-n t_{0}}{t_{0}-\tau} ; \quad n t_{0} \leq t<(n+1) t_{0}-\tau, \\
y_{1 \min }(t)\left(1-\frac{t-\left(n t_{0}-\tau\right)}{\tau}\right) ; n t_{0}-\tau \leq t<(n+1) t_{0},
\end{array}\right.
\end{aligned}
$$

where $n=0,1,2, \ldots$ corresponds to the consecutive laser-pulse period. In Fig. 4 the time dependencies of the spring-attachment position $y_{1}$ and its minimum value $y_{1 \min }$ are represented by solid and dashed curves, respectively. To avoid non-realistic discontinuities of the spring-attachment position's time derivative $\dot{y}_{1}$, the above-defined $y_{1}(t)$ time dependence was smoothed in order to take into account the rise time of the laser source $\tau_{\mathrm{ls}}=0.2 \mathrm{~ms}$ and the thermal penetration time $\tau_{\mathrm{tp}}$, estimated by $\left.\tau_{\mathrm{tp}}=\left(\mathrm{d}_{\mathrm{w}} / 2\right)^{2} /(\pi \alpha)\right)=0.85 \mathrm{~ms}$. For smoothing, a moving average filter was employed with a length of $0.135 t_{0}$, which is of the order of the thermal penetration time $\tau_{\mathrm{tp}}$ and resulted in the best agreement between the results of the model and the experiment.

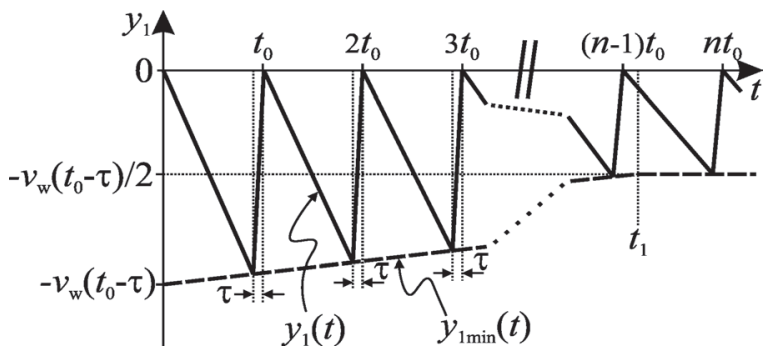

Fig. 4. Schematic presentation of the spring-attachment position's time dependence $y_{1}(t)$, the full curve; and its minimum value time dependence $y_{1 \min }(t)$, the dashed curve
The pendant droplet's mass time dependence $m(t)$ follows from the melting of the wire, as the melted material is incorporated into the pendant droplet. In the model, the wire is considered to melt whenever the spring-attachment position $y_{1}$, i.e., the droplet neck, travels downwards more slowly than the wire, which is fed by the constant feeding velocity $v_{\mathrm{w}}$. In this way, in addition to the melting of the wire by the laser pulse, melting of the wire between the laser pulses due to the droplet's internal energy is also taken into account. Thus, during an infinitesimally small time interval $d t$, the melted wire length $d l_{\mathrm{w}}$ can be expressed by a time derivative of the spring-attachment position $\dot{y}_{1}: d l_{\mathrm{w}}=\left(\dot{y}_{1}+v_{\mathrm{w}}\right) d t$. The corresponding pendant droplet's mass increase is then: $d m=A_{\mathrm{w}} \rho d l_{\mathrm{w}}=$ $A_{\mathrm{w}} \rho\left(\dot{y}_{1}+v_{\mathrm{w}}\right) d t$, where $A_{\mathrm{w}}=\pi d_{\mathrm{w}} 2 / 4$ is the crosssectional area of the wire, and $\rho$ is the density of the wire. The expression for $\mathrm{dm}$ is integrated in order to determine the pendant droplet's mass time dependence $m(t)$ :

$$
m(t)=m_{0}+\int_{0}^{t} A_{\mathrm{w}} \rho\left(\dot{y}_{1}+v_{\mathrm{w}}\right) d t
$$

where $m_{0}=m(t=0)$ is the pendant droplet's initial mass. Fig. 5 provides a schematic representation of the pendant droplet's mass time dependence $m(t)$, which corresponds to the spring-attachment position's time dependence $y_{1}(t)$, as presented in Fig. 4. From Fig. 5 it can be seen that at the beginning the mass of the pendant droplet increases only during the laser pulse of duration $\tau$. However, at later times, the mass slowly increases between the laser pulses due to melting of the wire by a large and hot pendant droplet. At all times, the droplet mass increase $\Delta m$ during one laserpulse period is equal to the mass of the wire that is fed during the same time: $\Delta m=A_{\mathrm{w}} \rho v_{\mathrm{w}} t_{0}$, as indicated in Fig. 5.

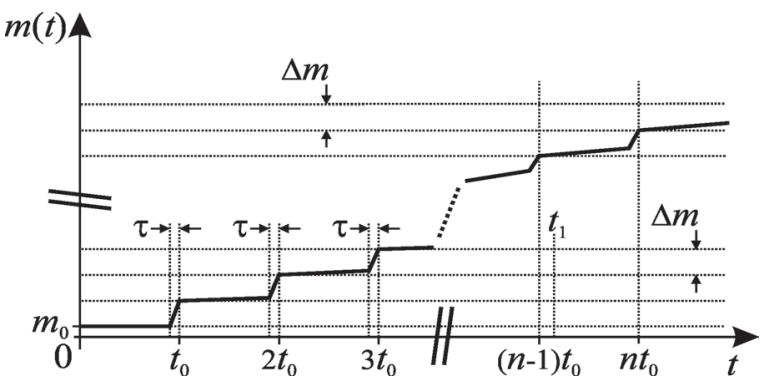

Fig. 5. Schematic presentation of pendant droplet's mass time dependence $m(t)$, corresponding to the spring-attachment position's time dependence $y_{1}(t)$

The length $l_{0}$ of the unloaded spring was presumed to be equal to the radius $d / 2$ of a spherical 
droplet, which is in accordance with the small gravitational Bond number $G$ and the experimental observations. The time dependence of the length $l_{0}$ was determined using the time dependence of the radius $d(t) / 2$, expressed by the droplet mass $m(t)$ and its density $\rho$ :

$$
l_{0}(t)=\frac{d(t)}{2}=\sqrt[3]{\frac{3 m(t)}{4 \pi \rho}} .
$$

The time-dependent spring stiffness $k(t)$ was determined from the experimental effective spring stiffness volume dependence $k(V)$ in Eq. (1) [16], by expressing the droplet volume $V(t)$ by its mass $m(t)$ and density $\rho$ :

$$
k(t)=k_{0}\left(\frac{m(t)}{\rho V_{0}}\right)^{a} .
$$

Since in previous work the value of the viscous damping coefficient was found to be too small to describe the damping of the metal pendant droplet, presumably due to rotational flow in the droplet, and, therefore, a constant value damping coefficient was used [18], the damping coefficient $c(t)$ of the proposed model was assumed to be constant. The value of $1.5 \times 10^{-3} \mathrm{~kg} / \mathrm{s}$ used in the calculation most closely reproduced the experimental droplet-oscillation amplitudes and detached-droplet diameters, and is similar to the value of $2.0 \times 10^{-3} \mathrm{~kg} / \mathrm{s}$, which was used in a similar model of a pendant steel droplet [18]. The employed value is indeed much larger than the viscous damping coefficient, estimated using the expression $3 \mu V /(d / 2)$ [19], which for a nickel droplet of diameter $d=1 \mathrm{~mm}$ gives $5.0 \times 10^{-5} \mathrm{~kg} / \mathrm{s}$. This indicates the important influence of other possible factors, such as rotational flow in the oscillating pendant droplet, driven by the temperature gradient, which are beyond the scope of this study.

The initial pendant droplet's mass $m(t=0)=m_{0}$ corresponds to the mass of the remaining pendant part after the detachment of the preceding droplet. The mass of the remaining pendant part was experimentally hard to estimate from the recorded infrared (IR) intensity images due to its small diameter and low temperature. For this reason, an initial pendant-droplet mass $m_{0}$ value was selected in the interval of less than $2.4 \times 10^{-7} \mathrm{~kg}$, which was experimentally estimated as the upper initial mass limit, and is of the order of the typical droplet massincrease $\Delta m$ during one laserpulse period.

In order to define the initial state of the model, the following initial values were selected. The initial spring-attachment position $y_{1}(t=0)$ was chosen to be
0 . The initial point-mass position $y(t=0)$ was then determined as its static force balance position: $y(t=0)=l_{0}(t=0)-m_{0} g / k(t=0)$. The initial point-mass velocity $\dot{y}_{1}$ was defined as being equal to the negative value of the wire-feeding velocity magnitude $\dot{y}_{1}(t=0)=-v_{\mathrm{w}}$. In this way, at $t=0$ the pendant droplet moves downwards with the wire.

The detachment of the pendant droplet was modelled using a dynamic force balance condition. For pendant droplet's detachment, the ratio $\eta$ of the combined inertial, $m \ddot{y}$, and gravitational, $m g$, forces to the maximum surface-tension force, $\pi d_{\mathrm{w}} \sigma$, which retains the pendant droplet attached to the wire, should exceed a value of one. Additionally, the point-mass velocity $\dot{y}$ should at the same time be directed downwards in order to avoid the case of detachment and immediate re-attachment of an upwards travelling droplet, which was observed experimentally [15]. Thus, the modelled pendant droplet detaches when both of the following conditions are fulfilled:

$$
\begin{aligned}
& \eta=\frac{m(\ddot{y}+g)}{\pi d_{\mathrm{w}} \sigma}>1, \\
& \dot{y}<0 .
\end{aligned}
$$

\section{RESULTS AND DISCUSSION}

The formulated model was solved using the RungeKutta numerical integration method at laser-pulse frequency $f_{\mathrm{p}}$ values between $60 \mathrm{~Hz}$ and $190 \mathrm{~Hz}$, using a step of $10 \mathrm{~Hz}$. At each frequency value, the model was solved for 31 equidistant values of the initial pendantdroplet mass $m_{0}$ from $0.04 \times 10^{-7} \mathrm{~kg}$ up to $2.4 \times 10^{-7} \mathrm{~kg}$, where the latter value was experimentally estimated as the upper initial mass limit.

In Fig. 6, examples of the modelled point-mass vertical position time series $y(t)$ are presented as thick red lines for laser-pulse frequency $f_{\mathrm{p}}$ values of $(70,100,140$, and 180$) \mathrm{Hz}$. The initial pendantdroplet mass $m_{0}$ was, in all cases, $0.748 \times 10^{-7} \mathrm{~kg}$. For comparison, the examples of the corresponding experimental pendant-droplet centroid vertical position time series $y(t)$ estimated from the high-speed IR records of the continuous ALDG process [16] are plotted in Fig. 6 using thin blue lines. In the presented cases the droplet detached is in resonance with $f_{\mathrm{p}}$. The moment of the detachment is denoted by a red square and a blue circle for the model and experiment, respectively.

As can be seen in Fig. 6, the model successfully reproduces the experimental vertical centroid position time series $y(t)$ except in some sections of 
the droplet growth, where an obvious discrepancy can be observed. The cause of this discrepancy is the pendulum-like lateral oscillation of the pendant droplet, which is revealed by the experimental horizontal centroid position time series $x(t)$, denoted by a dotted black line in Fig. 6. Indeed, at the time of the observed discrepancy, a large amplitude of lateral oscillation can be seen in the horizontal centroid position time series $x(t)$. Here, it should be noted that only a projection of the lateral oscillation on the IR camera image plane could be observed. In the case of a lateral oscillation perpendicular to the image plane, an oscillation in the $x$ coordinate would not be detected, while the lateral oscillation would still influence the detected vertical oscillation. The observed lateral oscillation could be induced by a small deviation from the axial symmetry, e.g., a small misalignment between the wire and the laser beam, and could result in large amplitudes in the resonance of one of the many oscillation modes of the pendant-droplet system, which is very similar to a spring pendulum [22]. Since the presented one-dimensional, mass-spring-damper model includes only the vertical oscillation, the lateral degree of freedom is unaccounted for, which probably led to the above-described discrepancy.

The modelled detachment time falls within $5 \%$ of the experimental detachment time, except at laser-pulse frequencies $f_{\mathrm{p}}$ above $150 \mathrm{~Hz}$, where the modelled detachment time shown in Fig. 6, $f_{\mathrm{p}}=180 \mathrm{~Hz}$, is much too small, which will be commented on at the end of the section. The good agreement between the modelled and experimental times of detachment is a consequence of the pendantdroplet resonance with the laser-pulse frequency $f_{\mathrm{p}}$, which provides both a large enough oscillation amplitude and a favorable phase difference of $\pi / 2$ between the pendant-droplet oscillation and the laser pulses [16]. The phenomenon is illustrated in Fig. 7,
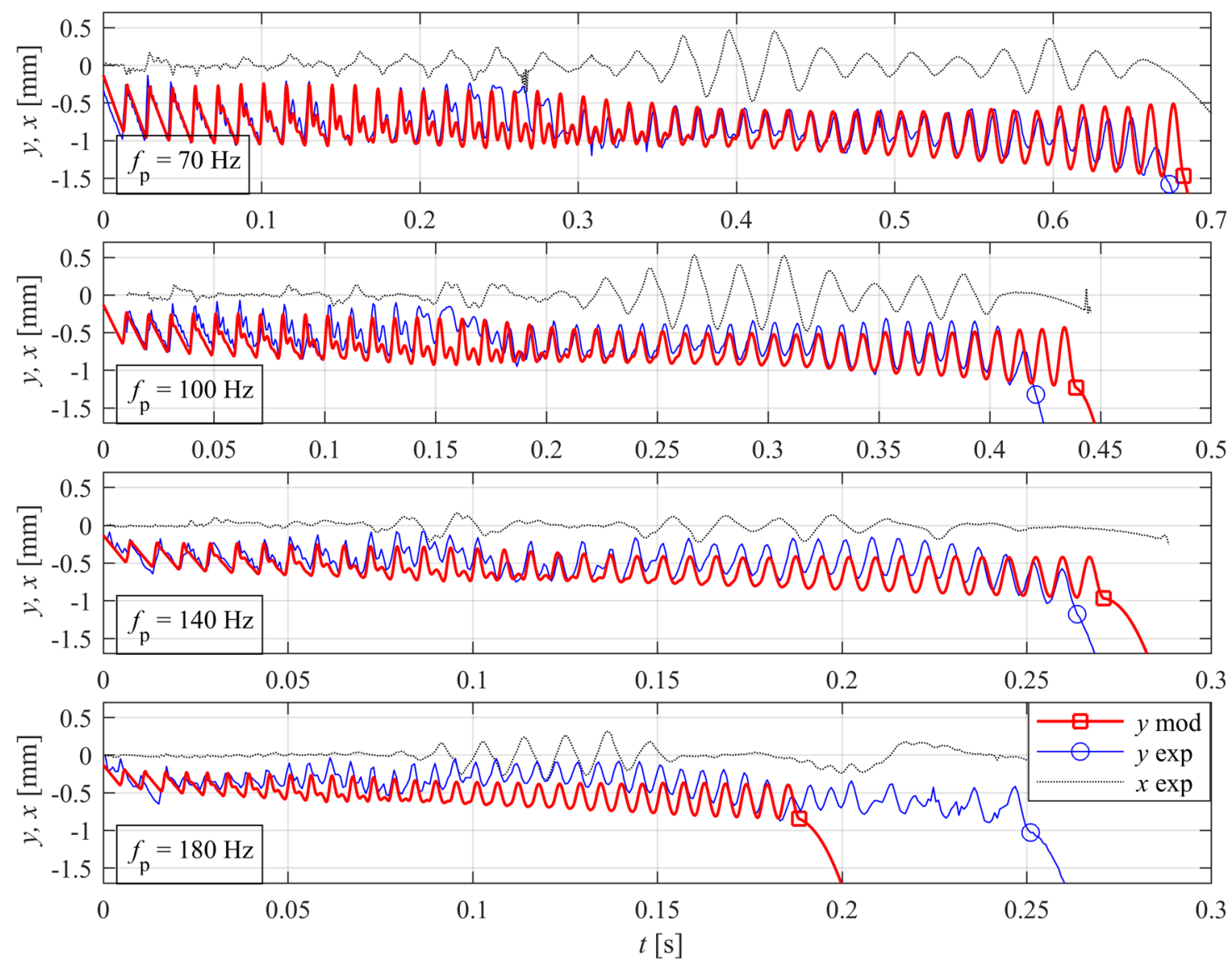

Fig. 6. Examples of the vertical centroid position time series $y(t)$ resulting from the model (marked by thick red lines, with small squares denoting the moment of detachment), and from the experiment (marked by thin blue lines,

with circles denoting the moment of detachment) with the experimentally determined horizontal centroid position time series $x(t)$ (marked by dotted black lines) of the droplets that detached in resonance with $f_{\mathrm{p}}$ 
where the harmonic-wavelet-based, time-frequency map [16] of the vertical position time series $y(t)$ is presented for the laser-pulse frequencies $f_{\mathrm{p}}=70 \mathrm{~Hz}$ and $180 \mathrm{~Hz}$ for both the modelled case (Fig. 7a)and the experimental case (Fig. 7b). In this figure, the small amplitudes are denoted by dark tones, whereas the large amplitudes are denoted by bright tones. The modelled and experimental cases presented in Fig. 7 show the same time evolution of the resonances at lower and lower multiples of the laser-pulse frequency $f_{\mathrm{p}}$, which is ended by detachment, denoted by a vertical dotted line, triggered by the resonance at the laser-pulse frequency $f_{\mathrm{p}}$. The detachment in all the presented cases takes place after the onset of the peak of the vertical oscillation amplitude, because only then does the phase difference between the droplet oscillation and the laser pulses reach a value close to $\pi / 2$ [16], when the inertial force is large enough and the detachment conditions are fulfilled. The nonmonotonically increasing amplitude at $f=f_{\mathrm{p}}$, which can be observed in the experimental time-frequency maps in Fig. 7b, follows from the vertical position $y(t)$ amplitude variation due to the lateral oscillation.

In the experiment as well as in the model, some smaller droplets were observed to detach at laser-pulse frequency $f_{\mathrm{p}}$ values below $120 \mathrm{~Hz}$. The detachment of these droplets was shown to coincide with the vertical oscillation resonance corresponding to the second multiple of the laser-pulse frequency $f_{\mathrm{p}}$ [16]. For droplets detached in this way at laser-pulse frequency $f_{\mathrm{p}}$ values of $70 \mathrm{~Hz}$ and $100 \mathrm{~Hz}$, examples of the model point-mass vertical position time series $y(t)$ are presented in Fig. 8 by means of a thick red line. The initial pendant-droplet mass $m_{0}$ was, in both cases, $0.512 \times 10^{-7} \mathrm{~kg}$. For comparison, the corresponding experimental pendant-droplet centroid vertical $y(t)$ and the horizontal $x(t)$ position time series are plotted by means of a thin blue line and a dotted black line, respectively. The moment of detachment is denoted by a small red square for the model and a blue circle for the experiment.

Again, the model successfully reproduced the experimental vertical centroid position time series $y(t)$, where the horizontal oscillation amplitude is low. It can be seen from Fig. 8 that, before detachment, as well as at the moment of detachment, the horizontal oscillation amplitude is high. It has been experimentally observed that the triggering of the detachment near the $2 f_{\mathrm{p}}$ resonance is due to the resonance of the oscillation mode combining vertical and lateral degrees of freedom [16]. Thus, the modelled time of detachment, where only the vertical oscillation is taken into account, is less similar to the experimental time of detachment. Also, the comparison of the corresponding time-frequency plots for the modelled case (Fig. 9a) and the experimental a)
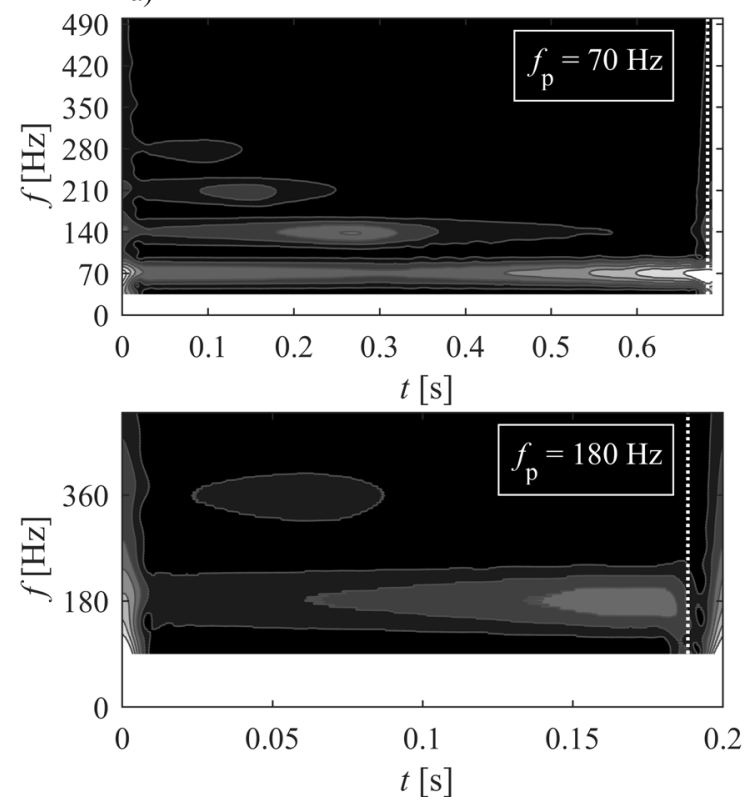

b)
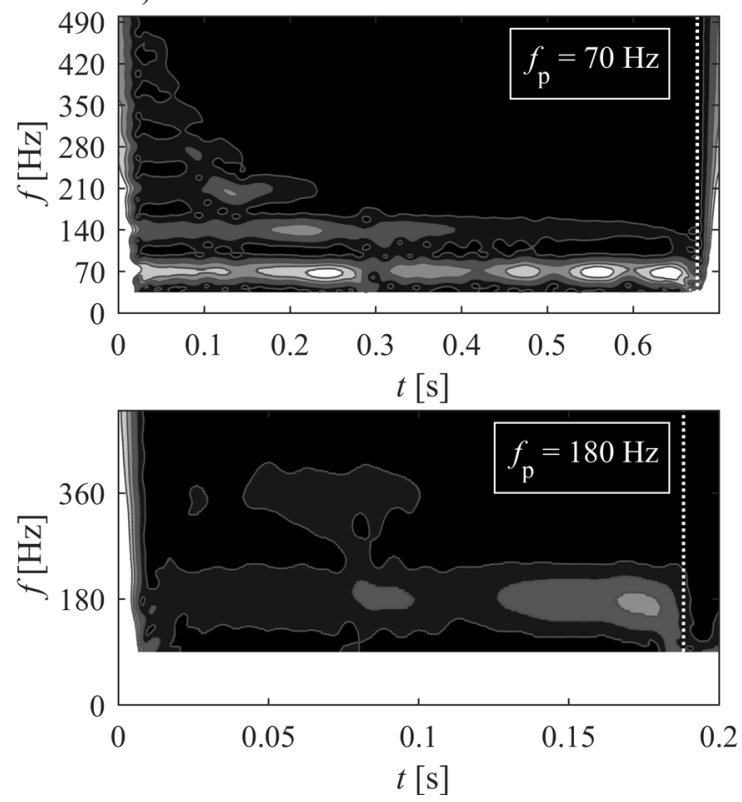

Fig. 7. Time-frequency maps of a) the modelled, and b) the experimental vertical centroid position time series $y(t)$ at $f_{\mathrm{p}^{\prime \prime}}=70 \mathrm{~Hz}$ and $180 \mathrm{~Hz}$ of the droplets that detached in resonance with $f_{\mathrm{p}}$ 

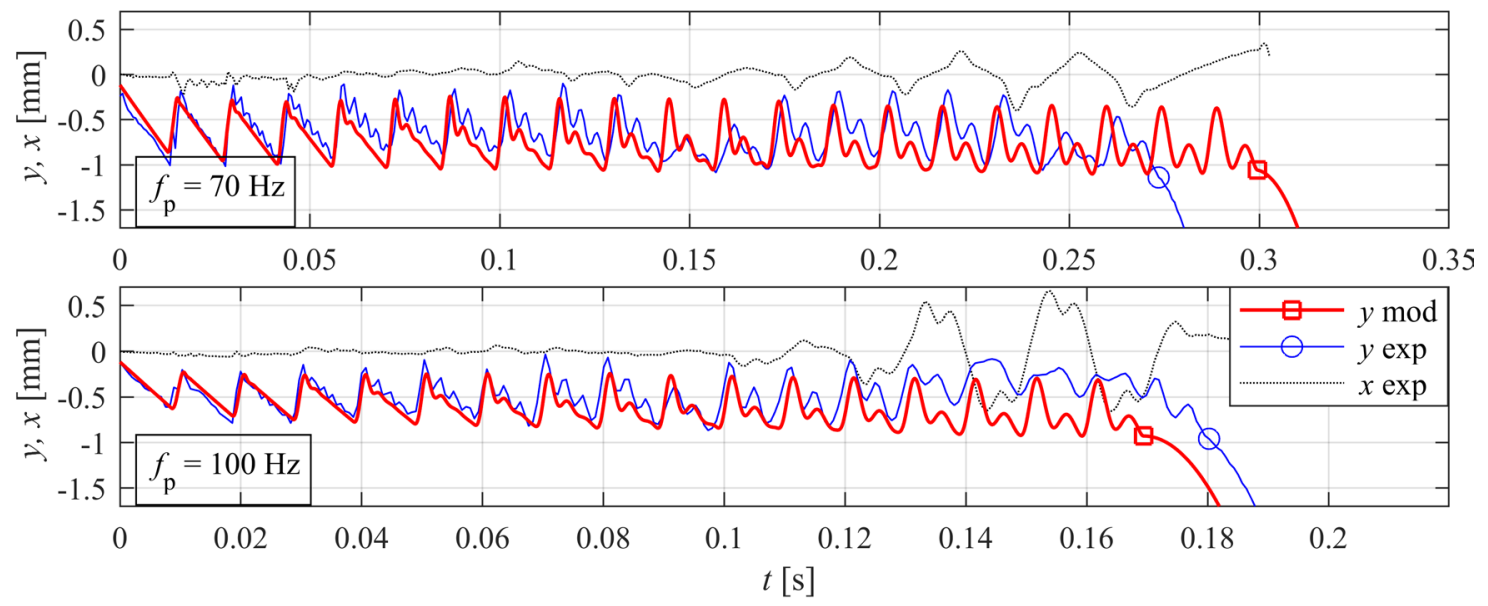

Fig. 8. Examples of vertical centroid position time series $y(t)$ resulting from the model (marked by a thick red line, with small squares denoting detachment) and the experiment (marked by a thin blue line, with small circles denoting the detachment) with the experimental horizontal centroid position time series $x(t)$ (shown by a dotted black line) of the droplets that detached in resonance with $2 f_{\mathrm{p}}$

a)

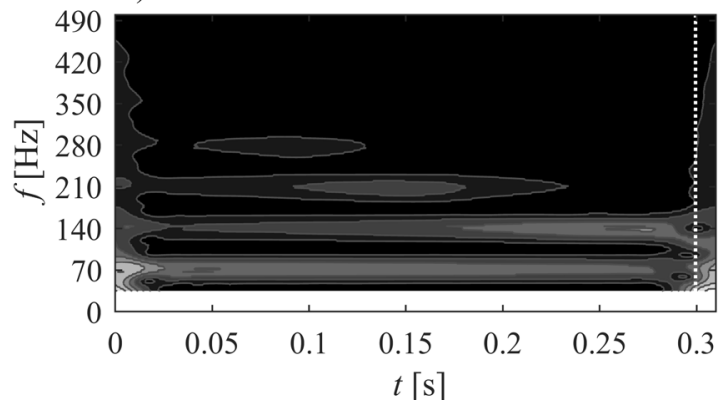

b)

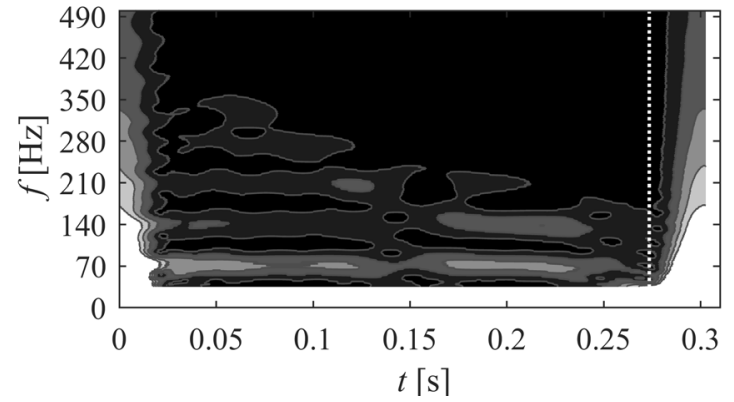

Fig. 9. Time-frequency maps of a) the modelled and b) the experimental vertical centroid position time series $y(t)$ at $f_{\mathrm{p}}=70 \mathrm{~Hz}$ of the droplets that detached in resonance with $2 f_{\mathrm{p}}$

case (Fig. 9b) at the laser-pulse frequency $f_{\mathrm{p}}=70 \mathrm{~Hz}$ reveals this difference. Whereas in the modelled case (Fig. 9a) the detachment, denoted by a vertical dotted line, takes place immediately after the resonant peak at $2 f_{\mathrm{p}}=140 \mathrm{~Hz}$, when the phase difference between the droplet oscillation and the laser pulses reaches a value close to $\pi / 2$, in the experimental case (Fig. 9b), the same peak is less pronounced, and the detachment is triggered later since it is a consequence of a resonance of the combined oscillation mode that includes both vertical and lateral degrees of freedom.

From the point of view of applications, the diameter $d_{\mathrm{d}}$ of the detached droplet is one of the most important output parameters of the droplet-generation process. Since the pendant-droplet detachment in continuous ALDG at laser-pulse frequency $f_{\mathrm{p}}$ values between $60 \mathrm{~Hz}$ and $190 \mathrm{~Hz}$ is triggered by resonance, the detached droplet diameter $d_{\mathrm{d}}$ depends on the laser-pulse frequency $f_{\mathrm{p}}$. In order to show this dependence, the modelled and experimental detacheddroplet diameters $d_{\mathrm{d}}$ are presented in Fig. 10 as small red circles and blue points, respectively, with their dependence on the laser-pulse frequency $f_{\mathrm{p}}$. As is evident from the figure, in the laser-pulse frequency $f_{\mathrm{p}}$ interval between $60 \mathrm{~Hz}$ and $150 \mathrm{~Hz}$, the model successfully reproduces the experimentally observed diameters, corresponding to the detachment triggered by the resonance at the laser-pulse frequency $f_{\mathrm{p}}$, denoted by the higher branch of blue points, and by the resonance at the second multiple $2 f$, denoted by the lower branch of blue points. Also, the bifurcation near $120 \mathrm{~Hz}$, below which the two branches coexist, was reproduced by the model.

In Fig. 10, the largest discrepancy between the modelled and the experimental values of the detacheddroplet diameter $d_{\mathrm{d}}$ can be observed at the lowest and at high values of the laser-pulse frequency $f_{\mathrm{p}}$. At the lowest frequency $f_{\mathrm{p}}=60 \mathrm{~Hz}$, the model predicts 
droplets with a diameter $d_{\mathrm{d}}$ between 1.2 and $1.3 \mathrm{~mm}$, whereas in the experiment the droplet diameter $d_{\mathrm{d}}$ shows much larger variations. The reason for this discrepancy is the influence of the Rayleigh-Plateau instability of the molten column of wire above the droplet, which at this low value of the laserpulse frequency $f_{\mathrm{p}}$ is long enough directly after the laser pulse to become unstable and can cause the detachment of a droplet with a smaller diameter [15]. On the other hand, at high values of the laser-pulse frequency, $f_{\mathrm{p}} \geq 150 \mathrm{~Hz}$, the modelled values of the detached droplet diameter $d_{\mathrm{d}}$ are clearly smaller than the experimentally observed values, which is also evident in Fig. 6 in the case of $f_{\mathrm{p}}=180 \mathrm{~Hz}$. Due to the high laser-pulse frequency, the vertical forcing peakto-peak amplitude $\left|y_{1 \text { min }}\right|$ is smaller, and the pendantdroplet detachment triggered by a vertical oscillation can be delayed due to the onset of the lateral oscillation, which slows down the rate of increase of the vertical oscillation amplitude. As can be seen in Fig. 10, up to the laser-pulse frequency $f_{\mathrm{p}}=180 \mathrm{~Hz}$, some experimental droplets were observed to detach with approximately the same diameter $d_{\mathrm{d}}$ (and at the same time) as the modelled ones. In these cases a lateral oscillation was not present. At the modelled laser-pulse frequency values $f_{\mathrm{p}} \geq 170 \mathrm{~Hz}$, the model predicts detached droplets with a diameter $d_{\mathrm{d}}$ near $1.0 \mathrm{~mm}$, whereas, in the experiment, also droplets with diameters greater than $2.0 \mathrm{~mm}$ were observed [15]. The reason for this discrepancy is the low vertical forcing amplitude combined with the lateral oscillation of the pendant droplet, which enable the pendant droplet to pass the resonance at $f_{\mathrm{p}}$ without detachment, so that it detaches later, having a larger diameter, due to the resonance of the $n=2$ mode [15].

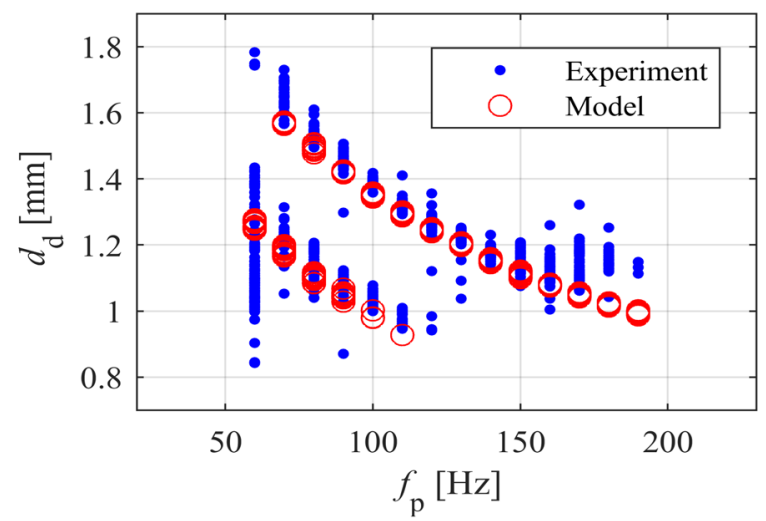

Fig. 10. Diameter $d_{\mathrm{d}}$ of the modelled and experimentally observed detached droplet in dependence on the laser pulse frequency $f_{\mathrm{p}}$

\section{CONCLUSIONS}

In the paper, the pendent-droplet oscillation and detachment in the continuous ALDG process were described by means of a mathematical, dynamic force balance, mass-spring-damper model in the laser-pulse frequency range between $60 \mathrm{~Hz}$ and $190 \mathrm{~Hz}$. The timedependent coefficients of the model were determined based on the experimental observations. The comparison between the modelled and experimental droplet centroid vertical position time series and their time-frequency maps show that the proposed model was able to reproduce the essential dynamics. The model also reproduced the pendant-droplet growth and detachment, triggered by the $n=1$ eigenmode resonance of the pendant droplet with the frequency of the laser pulses or its second multiple. Compared to the experimental results, the detached droplet diameter as a function of the laser-pulse frequency was predicted well by the model, including the bifurcation at $120 \mathrm{~Hz}$ and the coexistence of two detached-droplet diameter values at laser-pulse frequencies below 120 Hz. However, the modeled detached-droplet diameter differs from the experimental values at both ends of the selected laser-pulse frequency interval, where the droplet detachment is influenced by phenomena that are not accounted for by the model, but can be explained based on the experimental observations. This is because, at the lowest frequency, the droplet detachment is influenced by the Rayleigh-Plateau instability, whereas at the highest frequencies, the onset of the lateral pendulum-like oscillation can delay or even prevent the $n=1$ eigenmode resonant detachment. The description of the continuous ALDG could be improved by including the lateral degree of freedom in the presented model.

\section{ACKNOWLEDGEMENTS}

The authors acknowledge the financial support provided by the Slovenian Research Agency (Research core funding No. P2-0241).

\section{REFERENCES}

[1] Schmidt, M., Merklein, M., Bourell, D., Dimitrov, D., Hausotte, T., Wegener, K., Overmeyer, L., Vollertsen, F., Levy, G. N. (2017). Laser based additive manufacturing in industry and academia. CIRP Annals, vol. 66, no. 2, p. 561-583, DOl:10.1016/j.cirp.2017.05.011.

[2] Kotar, M., Govekar, E. (2018). The influence of the workpiece illumination proportion in annular laser beam wire deposition process. Procedia CIRP, vol. 74, p. 228-232, D0l:10.1016/j. procir.2018.08.100. 
[3] Mutiu, E.F., Akinlabi, E.T. (2018). Influence of laser power on improving the wear properties of laser-deposited Ti-6Al$4 \mathrm{~V}+\mathrm{B} 4 \mathrm{C}$ composite. Strojniški vestnik - Journal of Mechanical Engineering, vol. 64, no. 7-8, p. 488-495, Dol:10.5545/svjme.2018.5362.

[4] Govekar, E., Jerič, A., Weigl, M., Schmidt, M. (2009). Laser droplet generation: Application to droplet joining. CIRP Annals - Manufacturing Technology, vol. 58, no. 1, p. 205-208, D0I:10.1016/j.cirp.2009.03.005.

[5] Chen, C.-A., Chun, J.-H., Sohlenius, G. (1997). Development of a droplet-based manufacturing process for free-form fabrication. CIRP Annals, vol. 46, no. 1, p. 131-134, D0l:10.1016/S00078506(07)60791-4.

[6] Lee, T.-M., Kang, T.G., Yang, J.-S., Jo, J., Kim, K.-Y., Choi, B.O., Kim, D.-S. (2008). Drop-on-demand solder droplet jetting system for fabricating microstructure. IEEE Transactions on Electronics Packaging Manufacturing, vol. 31, no. 3, p. 202210, DOI:10.1109/TEPM.2008.926285.

[7] Cheng, S.X., Li, T., Chandra, S. (2005). Producing molten metal droplets with a pneumatic droplet-on-demand generator. Journal of Materials Processing Technology, vol. 159, no. 3, p. 295-302, D0I:10.1016/j.jmatprotec.2004.05.016.

[8] Sohn, H., Yang, D.Y. (2005). Drop-on-demand deposition of superheated metal droplets for selective infiltration manufacturing. Materials Science and Engineering: $A$, vol. 392, no. 1-2, p. 415-421, D0l:10.1016/J.msea.2004.09.049.

[9] Luo, Z., Wang, X., Wang, L., Sun, D., Li, Z. (2017). Dropon-demand electromagnetic printing of metallic droplets. Materials Letters, vol. 188, p. 184-187, D0l:10.1016/j. matlet.2016.11.021.

[10] Yi, H., Qi, L., Luo, J., Zhang, D., Li, H., Hou, X. (2018). Effect of the surface morphology of solidified droplet on remelting between neighboring aluminum droplets. International Journal of Machine Tools and Manufacture, vol. 130-131, p. 1-11, DOI:10.1016/j.jjmachtools.2018.03.006.

[11] Vega, E.J., Cabezas, M.G., Muñoz-Sánchez, B.N., Montanero, J.M., Gañán-Calvo, A.M. (2014). A novel technique to produce metallic microdrops for additive manufacturing. The International Journal of Advanced Manufacturing Technology, vol. 70, no. 5-8, p. 1395-1402, D0l:10.1007/s00170-0135357-3.
[12] Jeromen, A., Kuznetsov, A., Govekar, E. (2014). Laser droplet generation from a metal foil. Physics Procedia, vol. 56, p. 720729, DOI:10.1016/J.phpro.2014.08.079.

[13] Stein, S., Dobler, M., Radel, T., Strauß, M., Breitschwerdt, H., Hugger, F., Roth, S., Schmidt, M. (2017). Experimental and numerical investigations regarding laser drop on demand jetting of $\mathrm{Cu}$ alloys. Production Engineering, vol. 11, no. 3, p. 275-284, Dol:10.1007/s11740-017-0737-4.

[14] Govekar, E., Kuznetsov, A., Jerič, A. (2016). Drop on demand generation from a metal wire by means of an annular laser beam. Journal of Materials Processing Technology, vol. 227, p. 59-70, D0I:10.1016/J.jmatprotec.2015.07.026.

[15] Kuznetsov, A., Jeromen, A., Govekar, E. (2014). Droplet detachment regimes in annular laser beam droplet generation from a metal wire. CIRP Annals - Manufacturing Technology, vol. 63, no. 1, p. 225-228, D0l:10.1016/j.cirp.2014.03.051.

[16] Jeromen, A., Govekar, E. (2016). Time series analysis based study of a mass-spring-like oscillation and detachment of a metal pendant droplet. Mechanical Systems and Signal Processing, vol. 80, p. 503-516, D0l:10.1016/j. ymssp.2016.04.038.

[17] Watkins, A.D., Smartt, H.B., Johnson, J.A. (1992). A dynamic model of droplet growth and detachment in GMAW, Proceedings of $3^{\text {rd }}$ International Conference on Trends in Welding Research, Gatlingburg, p. 993-997.

[18] Jones, L.A., Eagar, T.W., Lang, J.H. (1998). A dynamic model of drops detaching from a gas metal arc welding electrode. Journal of Physics D: Applied Physics, vol. 31, no. 1, p. 107123, DOI:10.1088/0022-3727/31/1/014.

[19] Choi, J.H., Lee, J., Yoo, C.D. (2001). Dynamic force balance model for metal transfer analysis in arc welding. Journal of Physics D: Applied Physics, vol. 34, no. 17, p. 2658-2664, DOl:10.1088/0022-3727/34/17/313.

[20] Basaran, O.A., DePaoli, D.W. (1994). Nonlinear oscillations of pendant drops. Physics of Fluids, vol. 6, no. 9, p. 2923-2943, DOI:10.1063/1.868120.

[21] Liu, H. (2000). Science and Engineering of Droplets: Fundamentals and Applications, Noyes Publications, Park Ridge \& William Andrew Publishing, Norwich.

[22] van der Weele, J.P., de Kleine, E. (1996). The order-chaosorder sequence in the spring pendulum. Physica A: Statistical Mechanics and its Applications, vol. 228, no. 1-4, p. 245-272, DOI:10.1016/0378-4371(95)00426-2. 